\title{
BI-EMBEDDINGS OF GRAPHS
}

\author{
by I. ANDERSON and R. J. COOK
}

(Received 22 October, 1973)

Let $\gamma$ and $\gamma^{\prime}$ be non-negative integers. We say that the graph $G$ is $\left(\gamma, \gamma^{\prime}\right)$ bi-embeddable if $G$ can be embedded in a surface of genus $\gamma$ and the complement $\bar{G}$ of $G$ can be embedded in a surface of genus $\gamma^{\prime}$. Let $N\left(\gamma, \gamma^{\prime}\right)$ be the least integer such that every graph with at least $N\left(\gamma, \gamma^{\prime}\right)$ points is not $\left(\gamma, \gamma^{\prime}\right)$ bi-embeddable. It has been shown in [1] and [5] that $N(0,0)=9$; this result was also obtained by John R. Ball of the Carnegie Institute of Technology. Our object here is to obtain upper and lower bounds for $N\left(\gamma, \gamma^{\prime}\right)$.

Let $S_{p}$ denote the closed orientable 2-manifold of genus $p$. The genus $\gamma(G)$ of the graph $G$ is the minimum value of $p$ for which $G$ may be embedded in $S_{p}$. Youngs [7] has shown that for such a minimal embedding every face is a 2-cell, and so we may apply Euler's formula. If $\gamma \geqq \gamma(G)$, then clearly $G$ can be embedded in a surface of genus $\gamma$, although the faces need not be 2-cells.

THEOREM 1.

$$
N\left(\gamma, \gamma^{\prime}\right) \leqq 8+\sqrt{ }\left(18+12\left(\gamma+\gamma^{\prime}\right)\right) .
$$

Proof. Let the connected components of $G$ be $H_{1}, H_{2}, \ldots$. Let $\gamma_{i}$ be the genus of $H_{i}$ and suppose that $H_{i}$ has $p_{i}$ points, $q_{i}$ lines and $r_{i}$ faces. Since every face of $H_{i}$ has at least 3 lines on its boundary, we have $2 q_{i} \geqq 3 r_{i}$. Substituting this in Euler's formula, we obtain

so that

$$
2-2 \gamma_{i}=p_{i}-q_{i}+r_{i} \leqq p_{i}-q_{i} / 3,
$$

$$
q_{i} / p_{i} \leqq 3+6\left(\gamma_{i}-1\right) / p_{i} .
$$

The average degree $d\left(H_{i}\right)$ of the points of $H_{i}$ is $2 q_{i} / p_{i}$ and the average degree $d(G)$ of the points of $G$ is given by

$$
d(G)=p^{-1} \sum_{i} d\left(H_{i}\right) p_{i} \leqq p^{-1} \sum_{i} p_{i}\left(6+12\left(\gamma_{i}-1\right) / p_{i}\right),
$$

where $p$ is the number of points of $G$, so that $p=\sum_{i} p_{i}$. Thus

$$
d(G) \leqq 6+12 p^{-1} \sum_{i}\left(\gamma_{i}-1\right) .
$$

From Corollary 2 of [2] we have

$$
\gamma(G)=\sum_{i} \gamma\left(H_{i}\right),
$$

so that, if $G$ can be embedded in a surface of genus $\gamma$, then

$$
d(G) \leqq 6+12(\gamma-1) / p .
$$

Similarly, if $\bar{G}$ can be embedded in a surface of genus $\gamma^{\prime}$, then

$$
d(\bar{G}) \leqq 6+12\left(\gamma^{\prime}-1\right) / p .
$$


We now observe that $d(G)+d(\bar{G})=p-1$, so that, if $G$ is $\left(\gamma, \gamma^{\prime}\right)$ bi-embeddable, we have

which gives

$$
p-1 \leqq 12+12\left(\gamma+\gamma^{\prime}-2\right) / p \text {, }
$$

$$
p \leqq \frac{1}{2}\left(13+\sqrt{ }\left(73+48\left(\gamma+\gamma^{\prime}\right)\right)\right)
$$

Therefore

$$
N\left(\gamma, \gamma^{\prime}\right) \leqq \frac{1}{2}\left(15+\sqrt{(}\left(73+48\left(\gamma+\gamma^{\prime}\right)\right)\right) \leqq 8+\sqrt{ }\left(18+12\left(\gamma+\gamma^{\prime}\right)\right)
$$

which completes the proof.

Before considering lower bounds for $N\left(\gamma, \gamma^{\prime}\right)$, we observe that $N\left(\gamma, \gamma^{\prime}\right)=N\left(\gamma^{\prime}, \gamma\right)$; so we may suppose that $\gamma \geqq \gamma^{\prime}$.

THEOREM 2. If $\gamma \geqq \gamma^{\prime} \geqq 5 \gamma / 6$, then

$$
N\left(\gamma, \gamma^{\prime}\right) \geqq \sqrt{(}\left(8 \gamma+12 \gamma^{\prime}\right) .
$$

Proof. We put $h=\gamma /\left(\gamma+\gamma^{\prime}\right)$, so that $\frac{1}{2} \leqq h \leqq 6 / 11$. Let

$$
\begin{aligned}
m & =\sqrt{ }(3-h)+\sqrt{ }(3-5 h), & n & =\sqrt{ }(3-h)-\sqrt{ }(3-5 h), \\
x & =\left\{m \sqrt{ }\left(\gamma+\gamma^{\prime}\right)\right\}+1, & y & =\left\{n \sqrt{ }\left(\gamma+\gamma^{\prime}\right)\right\}+1,
\end{aligned}
$$

where $\{x\}$ is the least integer not less than $x$. We take $G$ to be the complete bipartite graph $K_{x, y}$. Using a result of Ringel [3], we see that

$$
\gamma(G)=\{(x-2)(y-2) / 4\} \leqq m n\left(\gamma+\gamma^{\prime}\right) / 4=\gamma .
$$

Also, $\bar{G}=K_{x} \cup K_{y}$; so, using the well-known result of Ringel and Youngs [4], we see that

$$
\gamma(\bar{G}) \leqq\{(x-3)(x-4) / 12\}+\{(y-3)(y-4) / 12\} \leqq\left(m^{2}+n^{2}\right)\left(\gamma+\gamma^{\prime}\right) / 12=(1-h)\left(\gamma+\gamma^{\prime}\right)=\gamma^{\prime} .
$$

Therefore $N\left(\gamma, \gamma^{\prime}\right)>x+y \geqq 2 \sqrt{ }(3-h) \sqrt{ }\left(\gamma+\gamma^{\prime}\right)=\sqrt{ }\left(8 \gamma+12 \gamma^{\prime}\right)$.

COROLlaRY 1. $\quad \sqrt{ }(20 \gamma) \leqq N(\gamma, \gamma) \leqq 8+\sqrt{ }(18+24 \gamma)$.

THEOREM 3. If $5 \gamma / 6 \geqq \gamma^{\prime} \geqq \frac{1}{2} \gamma$, then

$$
N\left(\gamma, \gamma^{\prime}\right) \geqq \sqrt{ }\left(8 \gamma+12 \gamma^{\prime}\right) .
$$

Proof. We put $m=\{\sqrt{ }(2 \gamma)\}$ and take $G$ to be the complete tripartite graph $K_{m, m, m}$ so that $\bar{G}=3 K_{m}$. From a theorem of Ringel and Youngs which has since been generalized by White [6], we see that

$$
\gamma(G)=\frac{1}{2}(m-2)(m-1)<\frac{1}{2}(m-1)^{2} \leqq \gamma .
$$

Using the estimate of Ringel and Youngs [4], we have

$$
\gamma(\bar{G}) \leqq 3\{(m-3)(m-4) / 12\} \leqq(m-1)^{2} / 4 \leqq \frac{1}{2} \gamma \leqq \gamma^{\prime} .
$$

Hence, $\quad N\left(\gamma, \gamma^{\prime}\right)>3 m=3\{\sqrt{ }(2 \gamma)\} \geqq \sqrt{ }(18 \gamma)$.

Theorem 3 is of particular interest when $\gamma^{\prime}=\frac{1}{2} \gamma$, since the coefficient of $\sqrt{ } \gamma$ is then best possible. 
Corollary 2.

$$
\sqrt{ }(18 \gamma) \leqq N\left(\gamma, \frac{1}{2} \gamma\right) \leqq 8+\sqrt{ }(18+18 \gamma) .
$$

THEOREM 4. If $\frac{1}{2} \gamma \geqq \gamma^{\prime} \geqq 0$, then

$$
N\left(\gamma, \gamma^{\prime}\right) \geqq 3+H(\gamma),
$$

where $H(\gamma)=\left[\frac{1}{2}(7+\sqrt{ }(1+48 \gamma))\right]$ and $[x]$ denotes the integer part of $x$.

Proof. Let $H=H(\gamma)$; from [4] we see that the complete graph $K_{H}$ can be embedded in a surface of genus $\gamma$. We take $G=K_{H} \cup K_{2}$. Since $K_{2}$ can be placed in one of the faces in the embedding of $K_{H}$ we see that $G$ may also be embedded in a surface of genus $\gamma$. Then $\bar{G}$ is the complete bipartite graph $K_{H, 2}$, which is planar. Hence

$$
N\left(\gamma, \gamma^{\prime}\right) \geqq N(\gamma, 0)>H+2,
$$

which completes the proof.

When $\gamma^{\prime}=0$, the coefficient of $\sqrt{ } \gamma$ in the lower bound is $\sqrt{ } 12$, which is best possible.

CoROLlaRy 3. $\quad\left[\frac{1}{2}(13+\sqrt{ }(1+48 \gamma)] \leqq N(\gamma, 0) \leqq 8+\sqrt{ }(18+12 \gamma)\right.$.

White [6] proved that the genus of the complete tripartite graph $K_{m n, n, n}$ is given by

$$
\gamma\left(K_{m n, n, n}\right)=\frac{1}{2}(m n-2)(n-1)<\frac{1}{2} m n^{2},
$$

and we shall use this to obtain lower bounds for $N\left(\gamma, \gamma^{\prime}\right)$ which improve on Theorem 4 when $\gamma^{\prime}$ is greater than a suitable multiple of $\gamma$.

THEORBM 5. Let $m \geqq 12$ be an integer; then, for $\gamma \geqq \gamma^{\prime} \geqq 6 m \gamma /\left(m^{2}+2\right)$, we have

$$
N\left(\gamma, \gamma^{\prime}\right) \geqq(m+2)\left[\left(m^{2}+2\right)^{-\frac{1}{2}} \sqrt{ }(12 \gamma)\right] .
$$

Proof. We put $n=\left[\sqrt{ }\left(12 \gamma /\left(m^{2}+2\right)\right)\right]$ and take $\bar{G}=K_{m n, n, n}$, so that

$$
\gamma(\bar{G})<\frac{1}{2} m n^{2} \leqq 6 m \gamma /\left(m^{2}+2\right) \leqq \gamma^{\prime} .
$$

Then $G=K_{m n} \cup 2 K_{n}$ so that

$$
\begin{aligned}
\gamma(G) & \leqq \\
& \leqq(m n-3)(m n-4) / 12\}+2\{(n-3)(n-4) / 12\} \\
& \left.m^{2}+2\right) n^{2} / 12 \leqq \gamma .
\end{aligned}
$$

Hence $N\left(\gamma, \gamma^{\prime}\right)>m n+2 n=(m+2)\left[\left(m^{2}+2\right)^{-\frac{1}{2}} \sqrt{ }(12 \gamma)\right]$.

Finally we remark that the cases $m=5$ and $m=6$ may be used to obtain the following improvements on Theorem 2. Since the details are similar to those in the proof of Theorem 5, the proofs are omitted.

COROLlaRY 4. If $\gamma \geqq \gamma^{\prime} \geqq 18 \gamma / 19$, then

$$
N\left(\gamma, \gamma^{\prime}\right) \geqq 8[\sqrt{ }(6 \gamma / 19)]>\sqrt{ }(20.2 \ldots \gamma) \text {, as } \gamma \rightarrow \infty .
$$

COROLlaRY 5. If $18 \gamma / 19 \geqq \gamma^{\prime} \geqq 9 \gamma / 10$, then

$$
N\left(\gamma, \gamma^{\prime}\right) \geqq 7[\sqrt{ }(2 \gamma / 5)] .
$$


Note added in proof. B. L. Garman of Western Michigan University has recently proved that $\gamma\left(K_{n, n, n, n}\right)=(n-1)^{2}$ when $n \equiv 2(\bmod 4)$. It follows from this result that $N\left(\gamma, \gamma^{\prime}\right) \geqq 4 \sqrt{ } \gamma$ for $\gamma^{\prime} \geqq \gamma / 3$, so that the bound of Theorem 1 is also best possible at $\gamma^{\prime}=\gamma / 3$.

\section{REFERENCES}

1. J. Battle, F. Harary and Y. Kodama, Every planar graph with nine points has a non-planar complement, Bull. Amer. Math. Soc. 68 (1962), 569-571.

2. J. Battle, F. Harary, Y. Kodama and J. W. T. Youngs, Additivity of the genus of a graph, Bull. Amer. Math. Soc. 68 (1962), 565-568.

3. G. Ringel, Das Geschlecht des vollständigen paaren Graphen, Abh. Math. Sem. Univ. Hamburg 28 (1965), 139-150.

4. G. Ringel and J. W. T. Youngs, Solution of the Heawood map-coloring problem, Proc. Nat. Acad. Sci. U.S.A. 60 (1968), 438-445.

5. W. T. Tutte, On the non-biplanar character of the complete 9-graph, Canad. Math. Bull. 6 (1963), 319-330.

6. A. T. White, The genus of the complete tripartite graph $K_{m n, n, n}, J$. Combinatorial Theory 7 (1969), 283-285.

7. J. W. T. Youngs, Minimal imbeddings and the genus of a graph, J. Math. Mech. 12 (1963), 303-315.

UNIVERSITY OF GLASGOW

University College CardifF

GLASGOW G12 8QW 